\title{
Fertility outcome after treatment of retained products of conception: a systematic review
}

Tjalina W. O. Hamerlynck ${ }^{1 *}$, Dora Meyers ${ }^{2}$, Hannelore Van der Veken ${ }^{1}$, Jan Bosteels ${ }^{1,3}$ and Steven Weyers ${ }^{1}$

\begin{abstract}
Background: Treatment of retained products of conception (RPOC) can be expectant, medical or operative. Surgical removal of RPOC may lead to intrauterine adhesions (IUA) and Asherman's syndrome.

Objective: To evaluate how treatment options for RPOC affect future fertility by means of a systematic review. Search strategy: MEDLINE, EMBASE, The Cochrane Library, and clinical trial registers were searched, and reference lists were scanned.

Selection criteria: Randomised controlled trials (RCT) comparing different treatment options for RPOC (conservative, medical or surgical treatment, including curettage and/or hysteroscopic techniques, with or without application of anti-adhesion therapy), in women of reproductive age, were eligible for inclusion.

Data collection and analysis: Reviewers independently performed data extraction and quality of evidence assessment. For dichotomous variables, results were presented as risk ratio (RR) with 95\% $\mathrm{Cl}$.

Main results: Two studies were included. Nonsignificant differences were observed between the use of an antiadhesion barrier gel versus no treatment after operative hysteroscopy in IUAs (RR 0.32, 95\% Cl 0.04 to 2.80, $P$ value $=0.30$ ) and clinical pregnancy (RR $2.22,95 \% \mathrm{Cl} 0.67$ to $7.42, P$ value $=0.19$ ), and between hysteroscopic morcellation versus loop resection in IUAs (RR $0.86,95 \% \mathrm{Cl} 0.06$ to $13.12, P$ value $=0.91$ ).

Conclusion: There is insufficient evidence on how different treatment options for RPOC affect future reproductive outcomes. Results from ongoing RCTs are needed to guide clinicians towards choosing the best treatment.
\end{abstract}

Keywords: Retained products of conception, Treatment, Fertility, Reproductive outcome, Systematic review

\section{Introduction}

Retained products of conception (RPOC) consist of intrauterine tissue that develops after conception and persists after miscarriage, termination of pregnancy, delivery or caesarean section [1]. The occurrence of RPOC is not rare; however, the prevalence varies widely (from 0.5 to as much as 19\%) depending on pregnancy duration, pregnancy outcome and the successive management [2]. There is no consensus in literature on the type of tissue RPOC comprise. Some authors describe RPOC as non-villous trophoblastic tissue, chorionic villi or foetal membranes, and they state that decidua alone does not fall within the

\footnotetext{
*Correspondence: tjalina.hamerlynck@ugent.be

'Women's Clinic, Ghent University Hospital, C. Heymanslaan 10, 9000 Ghent, Belgium

Full list of author information is available at the end of the article
}

definition [3-5]. Others say that RPOC consist of the gestational sac, the decidua capsularis, chorionic villi or the embryo itself [6]. Still others argue that RPOC are from placental origin, and thus, that the presence of chorionic villi in RPOC is necessary $[1,7]$.

The diagnosis of RPOC remains a clinical challenge. The existence of RPOC may be suspected based on clinical history, with patients having symptoms of vaginal bleeding, abdominal or pelvic pain, and/or fever. However, RPOC may also be present in asymptomatic patients [8]. Ultrasound (US) findings such as a thickened endometrial echo complex or the presence of an endometrial mass with or without detectable vascularity at colour or power Doppler US are highly suggestive for RPOC [1]. Still, there is no consensus on the US criteria, and US alone may not be sensitive and/or specific 
enough to confirm the presence or absence of RPOC [1]. In order to avoid unnecessary procedures, diagnostic hysteroscopy may be of additional value to ultrasonography, although this needs further research $[9,10]$.

In case expectant management or medical treatment of RPOC fails, the surgical treatment traditionally consists of dilation and curettage, preferably under US guidance, using vacuum aspiration and/or a metal curette. Nevertheless, operative hysteroscopy is a suitable alternative to 'blind' curettage in the treatment of RPOC [5, 11-16]. These surgical procedures for RPOC, however, expose the uterus to additional trauma, which can cause intrauterine adhesions (IUAs) and Asherman's syndrome, clinically manifested by menstrual abnormalities, infertility and recurrent pregnancy loss [17].

The objective of the present study was to evaluate how the different treatment options for RPOC affect future fertility.

\section{Methods}

We specified the methods in advance and registered the protocol of the review on PROSPERO (CRD42016042444). We followed the PRISMA guidelines for writing a systematic review.

\section{Criteria for selecting studies for this review Types of studies}

Published parallel-group randomised controlled trials (RCTs) were eligible for inclusion. Non-randomised studies (e.g. studies with evidence of inadequate sequence generation such as alternate days, participant numbers) were excluded, as they are associated with a high risk of bias.

\section{Types of participants}

Women of reproductive age with RPOC more than $24 \mathrm{~h}$ after end of pregnancy, without the presence of gestational trophoblastic disease or uterine malignancies. Trials that excluded women who wished to conceive were not eligible.

\section{Types of interventions}

We included the following randomly assigned comparisons:

- Expectant management versus medical treatment

- Expectant management versus surgical removal by curettage or hysteroscopy

- Medical treatment versus surgical removal by curettage or hysteroscopy

- Curettage versus hysteroscopy

- Technique A versus technique B for operative hysteroscopy

- Anti-adhesion therapy versus placebo or no treatment following surgical treatment
The last two comparisons were not pre-defined in our review protocol. We included these randomised comparisons because at present different hysteroscopic techniques are available for the treatment of RPOC and anti-adhesion therapy following surgical treatment of RPOC may affect subsequent reproductive outcome [18]. The findings of our review might be biased if these two comparisons were omitted.

\section{Types of outcome measures}

Primary outcomes:

- Live birth rate. Live birth was defined as the complete expulsion or extraction from its mother of a product of fertilisation, irrespective of the duration of the pregnancy, which, after such separation, breathes or shows any other evidence of life, such as heart beat, umbilical cord pulsation, or definite movement of voluntary muscles, irrespective of whether the umbilical cord has been cut or the placenta is attached. We count the delivery of singleton, twin or multiple pregnancies as one live birth.

- Presence of IUAs at second-look hysteroscopy.

Secondary outcomes:

- Time to conception, conception rate and clinical pregnancy rate. Clinical pregnancy was defined as a pregnancy diagnosed by ultrasonographic visualisation of one or more gestational sacs or definitive clinical signs of pregnancy. It includes ectopic pregnancy. Multiple gestational sacs are counted as one clinical pregnancy.

- Miscarriage rate. Miscarriage was defined as the spontaneous loss of a clinical pregnancy before 20 completed weeks of gestational age (18 weeks after fertilisation) or, if gestational age is unknown, the loss of an embryo/foetus of less than $400 \mathrm{~g}$.

Eligible studies that could have measured the outcomes of interest were reviewed and any lack of data for the key outcomes was reported in the final review. We adhered as much as possible to terminology of the International Committee for Monitoring Assisted Reproductive Technology (ICMART) (http://www.icmartivf.org/) for key reproductive outcomes (live birth, pregnancy and miscarriage) [19]. We contacted primary study authors for clarification in cases of unclear definitions. We reported discrepancies or uncertainties in the final review.

\section{Search methods for identification of studies}

The following electronic bibliographic databases were searched: MEDLINE, EMBASE, and The Cochrane 
Library (Cochrane Central Register of Controlled Trials (CENTRAL)). The search strategy included terms relating to or describing the disease (retained products of conception), management or intervention (expectant, medical or surgical) and outcome (fertility). The search terms were adapted for use to each bibliographic database, and a combination of both $\mathrm{MeSH} /$ Emtree and free-text terms was used (Additional file 1: Appendix S1). There were no language restrictions in the search, but we included only articles in English, French, German, Dutch, Italian and Portuguese, due to restraints in time and cost. In addition, the reference lists of eligible studies were scanned, and clinical trial registers were searched for ongoing and registered trials (World Health Organization (WHO) International Clinical Trials Registry Platform (ICTRP) search portal (http://apps.who.int/trialsearch/) and ClinicalTrials.gov (https://clinicaltrials.gov)).

\section{Data collection and analysis \\ Selection of studies}

Duplicates of the studies obtained by the search strategy were removed using specialised software (EndNote X7). The titles and abstracts of the remaining studies were screened independently and simultaneously by two review authors (D.M., H.V.d.V.). The full texts of the potentially eligible studies were retrieved and independently assessed by the same two review team members for compliance with the inclusion criteria, and studies eligible for inclusion in the review were selected. Any disagreement during the selection was resolved through discussion or, if required, by consulting a third and/or fourth review author (T.H., S.W.).

\section{Data extraction and management}

A pre-piloted form (Additional file 2: Appendix S2) was used by two review authors (T.H., H.V.d.V.) independently to extract data from the included studies for assessment of study quality and evidence synthesis. Discrepancies were identified and resolved through discussion, with a third and/or fourth author (J.B., S.W.) where necessary. When information regarding an essential topic was missing or unclear, contact with the authors was attempted.

\section{Assessment of risk of bias in included studies}

Risk of bias of the included studies was independently assessed by two authors (T.H., H.V.d.V.) according to the Cochrane risk of bias assessment (random sequence generation, allocation concealment, blinding of participants and personnel, blinding of outcome assessment, incomplete outcome data, selective outcome reporting and other potential sources of bias) [20]. Disagreements between the review authors over the risk of bias in particular studies were resolved by discussion, with involvement of a third review author (J.B.) where necessary.

\section{Measures of treatment effect}

For dichotomous outcomes, we used risk ratios (RR) with $95 \%$ confidence intervals (CI).

\section{Data synthesis}

Statistical analysis was performed using the software REVIEW MANAGER 5.3 provided by the Cochrane Collaboration [21]. For dichotomous variables, results were presented as RR with 95\% CI. We aimed to perform statistical pooling if enough studies were retrieved. However, in case of clinical diversity or evidence of substantial statistical heterogeneity, we provided a narrative synthesis of the findings rather than statistical pooling.

\section{Results}

\section{Description of studies}

A total of 777 citations were identified from searching electronic databases and trial registers, of which $185 \mathrm{du}-$ plicate citations were removed. The remaining 592 records were assessed for eligibility through checking the titles and/or abstracts. We excluded 567 records as being obviously irrelevant. The eligibility of the remaining 25 articles was assessed by reading the full text. We retrieved six potentially eligible studies, we included two randomised trials (Additional file 3: Table S1), one was excluded (Additional file 4: Table S2) and three studies are ongoing (NCT02201732, NTR4923, ChiCTR-INR-16009 074) (Additional file 5: Table S3). The PRISMA flow chart for study selection is shown in Fig. 1.

We included two parallel-design RCTs [10, 22]. The first trial was performed in a tertiary medical care centre in Israel [22]. Fifty-two women underwent hysteroscopic surgery because of suspected RPOC. The mean age was 29.5 years (standard deviation (SD) 5.1 years) in the intervention group and 31.4 years (SD 6.5 years) in the control group. The trial did not include women with primary subfertility. After hysteroscopic removal of RPOC, a viscoelastic gel, composed of polyethylene oxide and carboxymethylcellulose, was applied or not. All patients received postoperative sequential hormone treatment and antibiotics. The outcomes were clinical pregnancy and presence of IUAs. The second trial was a multicentre study from Belgium and the Netherlands [10]. Eighty-six women who underwent hysteroscopic surgery because of RPOC were included. The mean age was 32 years (SD 6 years) in the hysteroscopic morcellation group and 31 years (SD 4 years) in the loop resection group. RPOC were removed by hysteroscopic morcellation or loop resection. In the resection group, cold loop resection was attempted first, whereas the loop was electrically activated in case the RPOC were too adherent to 


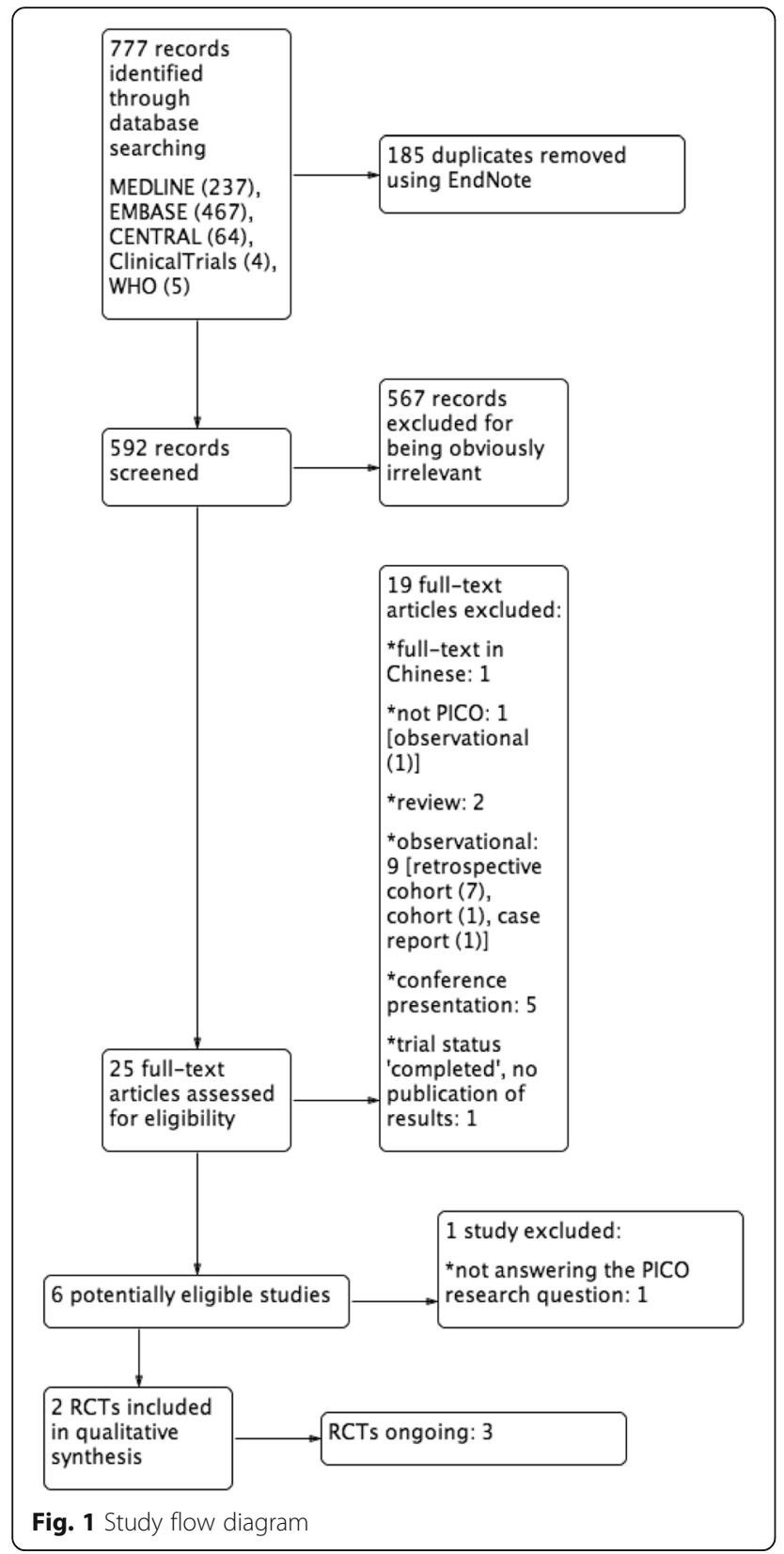

be removed by cold loop (4/39 cases, 10\%). Patients did not receive any cervical ripening agents or standard antibiotic prophylaxis preoperatively. The outcome was the presence of IUAs.

\section{Risk of bias in included studies}

See: assessment of the risk of bias (Additional file 6: Table S4) and Fig. 2. We judged both trials to be at low risk for selection bias related to random sequence generation and allocation, because a computer-generated randomisation list and sequentially numbered opaque sealed envelopes were used. We judged both trials to be at low risk of performance and detection bias in relation

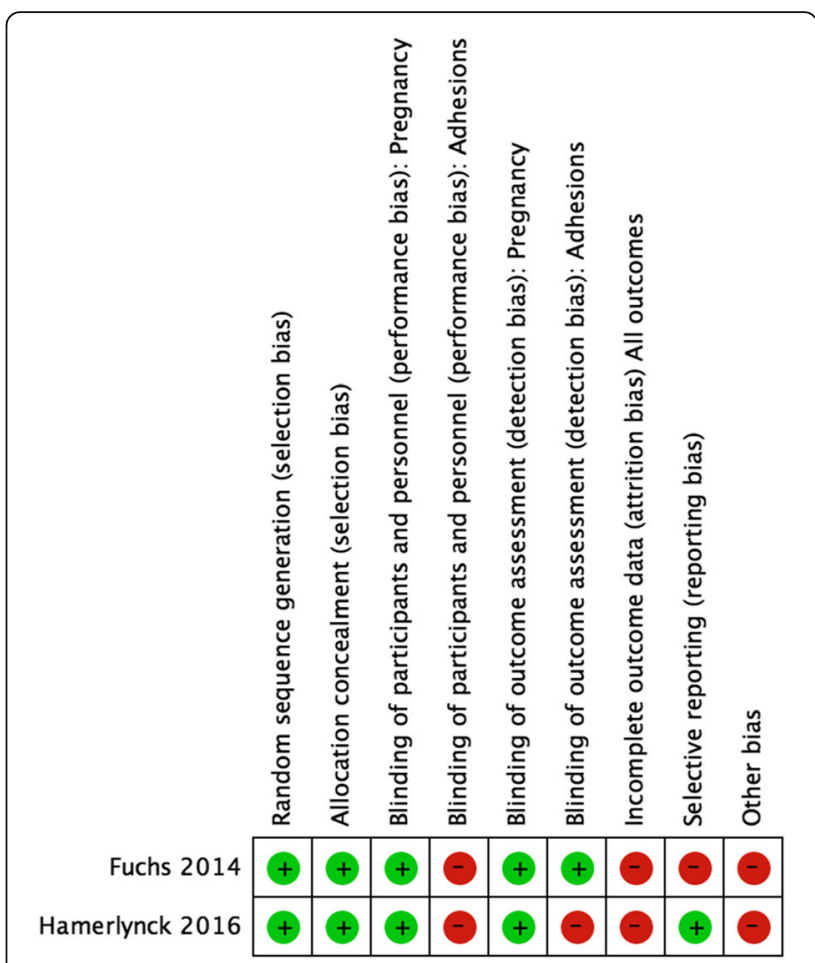

Fig. 2 Risk of bias summary

to blinding of participants, personnel and outcome assessors for the outcome of clinical pregnancy, because clinical pregnancy is an unequivocal outcome. For the outcome of presence of IUAs, we considered both trials to be at high risk of performance bias in relation to blinding of participants and personnel, because personnel were not blinded in the trial of Fuchs et al. and participants and personnel were not blinded in the trial of Hamerlynck et al. We found the trial of Fuchs et al. to be at low risk of detection bias for the key outcome of IUAs because outcome assessors were independent observers blinded to treatment allocation. However, we found the trial of Hamerlynck et al. to be at high risk of detection bias for the outcome of IUAs because outcome assessors were aware of the treatment allocation. We judged the trial of Fuchs et al. to be at high risk of attrition bias, because a high proportion of women were excluded after randomisation (11/52 or $21 \%$ ) without clarification of the reasons. Similarly, in the trial of Hamerlynck et al., 9 of 44 participants and 6 of 39 participants undergoing hysteroscopic removal of placental remnants did not undergo second-look hysteroscopy $(15 / 83$ or $18 \%)$, leading to a high risk of attrition bias. We considered the trial of Fuchs et al. to be at high risk of selective reporting, because it failed to report data for the primary outcome of live birth despite a study duration of 27 months. The trial of Hamerlynck et al. was considered to be at low risk of selective reporting, 
because all pre-specified outcomes were reported. We found the trial of Fuchs et al. to be at high risk of other potential sources of bias, because at follow-up hysteroscopy co-treatment with hysteroscopic adhesiolysis was offered to women with AFS II or III IUAs. The differences in co-treatment between both comparison groups-three of 20 (14\%) women in the control group and one of $21(4 \%)$ women in the intervention groupmay have affected the extent and course of the treatment effect. Similarly, in the trial of Hamerlynck et al., adhesiolysis was offered to women with IUAs, leading to a potential source of bias in relation to fertility outcome.

\section{Effects of interventions}

We retrieved no randomised studies for the comparisons of expectant management versus medical treatment or surgical removal, medical treatment versus surgical removal, or curettage versus hysteroscopy for the treatment of RPOC.

We found one randomised trial comparing anti-adhesion therapy with no treatment following surgical treatment [22]. There was no evidence of a statistically significant difference between both comparison groups for the outcome of IUAs at second-look hysteroscopy at 5 to 8 weeks (RR $0.32,95 \%$ CI 0.04 to $2.80, P$ value $=0.30$, one study, 41 women; Fig. 3). There were no statistically significant differences in clinical pregnancy rates between both comparison groups (RR 2.22, 95\% CI 0.67 to $7.42, P$ value $=0.19,1$ study, 41 women; Fig. 3 ). We retrieved one randomised trial comparing hysteroscopic morcellation with loop resection for removal of RPOC [10]. There was no evidence of a statistically significant difference between both comparison groups for the outcome of de novo IUAs at second-look hysteroscopy at 6 to 8 weeks (RR $0.86,95 \%$ CI 0.06 to $13.12, P$ value $=0.91,1$ study, 65 women; Fig. 4).

\section{Discussion}

\section{Main findings}

Our systematic review aimed at investigating the influence of the treatment of RPOC on reproductive outcome and the presence of IUAs. We searched for randomised trials comparing different treatment options (expectant management, medical and surgical treatment) for women of reproductive age with RPOC more than $24 \mathrm{~h}$ after end of pregnancy, in relation to fertility and/ or the presence of IUAs.

We found only one randomised study containing 41 participants on the use of an anti-adhesion barrier gel versus no treatment after hysteroscopic treatment of RPOC and one randomised study containing 86 participants on hysteroscopic morcellation versus loop resection for removal of RPOC [10, 22]. Both studies had a high risk of bias on four out of seven items. According to the results of both studies, there is no evidence of statistically significant differences between both comparison groups for the outcomes of clinical pregnancy or the presence of IUAs at second-look hysteroscopy.

\section{Strengths and limitations}

We aimed to follow the guidelines recommended by the Cochrane Handbook as much as possible [20]. We excluded non-randomised studies to minimise bias. We aimed at investigating the influence of the treatment of RPOC on fertility. Selection of studies and extraction of data was performed by two review authors independently.

Only two relevant randomised studies were identified. The studies were at high risk of bias, so results should be interpreted with caution, and they did not report on the primary outcome of live birth. However, for the trial performed by our group, we are considering follow-up of reproductive outcome for the patients as randomised [10]. Moreover, in the trial that did report on pregnancy, relevant information was lacking regarding the histologic

1.1 Presence of intrauterine adhesions at second-look hysteroscopy

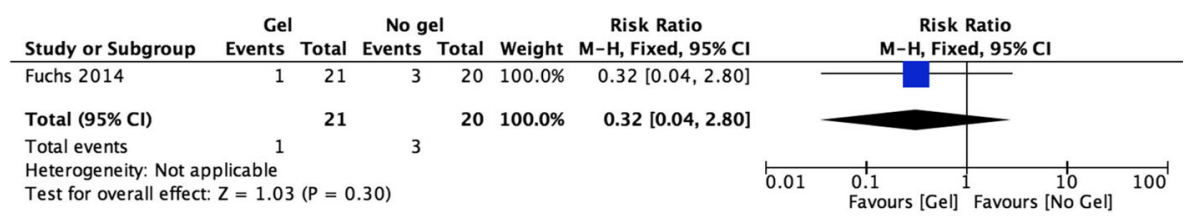

1.2 Clinical pregnancy

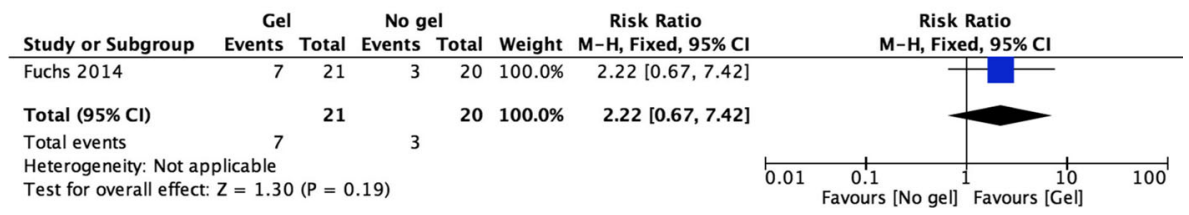

Fig. 3 Forest plot of comparison: 1 gel versus no treatment 
2.1 Presence of intrauterine adhesions at second-look hysteroscopy

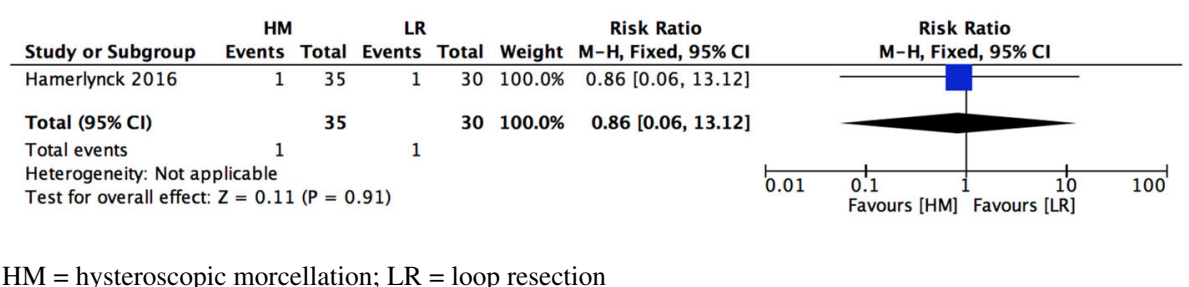

$\mathrm{HM}=$ hysteroscopic morcellation; $\mathrm{LR}=$ loop resection

Fig. 4 Forest plot of comparison: 2 hysteroscopic morcellation versus loop resection

criteria for RPOC, absolute numbers of pathologic confirmation of RPOC per group, the relation between histologic confirmation of RPOC and/or IUAs and/or fertility, as well as the number of women actually trying to reconceive after treatment of RPOC [22]. Our query related to these topics remained unanswered by the authors.

\section{Interpretation}

Previous reviews have addressed the topic of reproductive outcome after treatment of RPOC [23, 24]. Both reviews identified only cohort studies and no randomised trials. The trials included in the reviews studied the treatment of RPOC by curettage, hysteroscopy or both. The results in relation to IUAs appear to be in favour of hysteroscopic treatment of RPOC; however, no significant differences in conception or ongoing pregnancy rates were demonstrated. Due to the low methodological quality and high risk of bias in the included cohort studies-although a formal assessment of the risk of bias was lacking in both reviews-, the results need to be interpreted with caution. Our review was limited to randomised trials in order to reduce the risk of bias. We could identify one randomised trial examining the use of an anti-adhesion barrier after operative hysteroscopy for RPOC [22]. The study did not show a significant difference in the presence of IUAs or clinical pregnancy rate between intervention and control group. This finding is in line with the results of the systematic review of Bosteels et al. stating that the effectiveness of anti-adhesion therapy in improving reproductive outcome or reducing IUAs following operative hysteroscopy in subfertile women is still unclear [18]. The second study identified was a randomised trial comparing two hysteroscopic techniques, namely, hysteroscopic morcellation with loop resection for removal of RPOC [10]. The trial did not show a significant difference in the presence of IUAs. However, both identified trials may have been too small to demonstrate a difference in the outcome measures, and other factors than the techniques under study may have contributed to the risk of IUA formation or influenced the reproductive outcome.

\section{Conclusions}

\section{Implication for practice}

For daily clinical practice, the effectiveness of anti-adhesion treatment in improving key reproductive outcomes or decreasing IUAs following operative hysteroscopy for RPOC in women wishing to conceive remains uncertain. Similarly, it is not clear whether hysteroscopic morcellation or loop resection of RPOC leads to better reproductive outcomes or a lower risk of IUAs.

\section{Implication for research}

Additional studies are needed to evaluate the influence of RPOC on fertility. Hypothetically, there may be causal mechanisms linking the risk for RPOC with adverse reproductive outcomes such as infertility and recurrent miscarriage. The diagnostic modalities for RPOC need to be evaluated, and histopathological definitions need to be reviewed, aiming at reaching a consensus and providing practical guidelines. Different treatment modalities including expectant management, medical and surgical treatment (curettage, different hysteroscopic techniques) need to be compared to study reproductive outcome and cost-effectiveness. The size of the study population needs to be sufficiently large, and follow-up should be long enough to study the outcomes of interest. Obstetric history and other factors related to the occurrence of RPOC as well as the development of IUAs need to be considered when performing research on the subject. Our RCT, included in this review, should be considered as a pilot trial for hypothesis testing. Our future research agenda includes a pragmatic multicentre trial studying IUAs and reproductive outcome after surgical treatment of RPOC (NTR4923). Hysteroscopic morcellation (TRUCLEAR) is compared with US guided electric vacuum aspiration, because the latter is still the most frequently applied treatment for RPOC in Belgium and the Netherlands. There are currently two other ongoing trials examining following comparisons for treatment of RPOC: operative hysteroscopy (resection) versus vacuum aspiration (NCT02201732; primary outcome: intrauterine pregnancy lasting up to at least 22 weeks of gestation), and hysteroscopic morcellation (MyoSure) versus hysteroscopic 
monopolar loop resection (ChiCTR-INR-16009074; secondary outcomes: IUAs and pregnancy rate).

\section{Additional files}

Additional file 1: Appendix S1. Search strategy for systematic review on fertility outcome after treatment of retained products of conception. (109 kb)

Additional file 2: Appendix S2. Items of the pilot-tested data extraction form. (DOCX 52 kb)

Additional file 3: Table S1. Characteristics of included studies. (DOCX $133 \mathrm{~kb}$ )

Additional file 4: Table S2. Characteristics of excluded studies. (DOCX $45 \mathrm{~kb}$ )

Additional file 5: Table S3. Characteristics of ongoing studies. (DOCX $100 \mathrm{~kb}$ )

Additional file 6: Table S4. Risk of bias table. (DOCX 89 kb)

\section{Abbreviations}

ICTRP: International Clinical Trials Registry Platform; IUA: Intrauterine adhesion; RCT: Randomised controlled trial; RPOC: Retained products of conception; RR: Risk ratio; SD: Standard deviation; US: Ultrasound; WHO: World Health Organization

\section{Acknowledgements}

We thank Mrs. De Sutter, reference librarian KCGG of the Ghent University, for her assistance with the search strategy.

\section{Capsule}

There is insufficient evidence on how different treatments for retained products of conception affect future fertility.

\section{Authors' contributions}

$\mathrm{TH}$ took the lead in writing the manuscript. TH, DM, HVdV and SW contributed to the conception and design, as well as the acquisition of data. $\mathrm{TH}, \mathrm{DM}, \mathrm{HVdV}, \mathrm{JB}$ and SW contributed to the analysis and interpretation of data, drafting, revising and final approval of the review.

\section{Ethics approval and consent to participate}

Not applicable.

\section{Consent for publication}

Not applicable.

\section{Competing interests}

T.H. received a Clinical PhD Fellowship grant from the Research FoundationFlanders (FWO).

\section{Publisher's Note}

Springer Nature remains neutral with regard to jurisdictional claims in published maps and institutional affiliations.

\section{Author details}

'Women's Clinic, Ghent University Hospital, C. Heymanslaan 10, 9000 Ghent, Belgium. ${ }^{2}$ Faculty of Medicine and Health Sciences, Ghent University, C. Heymanslaan 10, 9000 Ghent, Belgium. ${ }^{3}$ Department of Gynaecology, Imelda Hospital, Imeldalaan 9, 2820, Bonheiden, Belgium.

Received: 12 February 2018 Accepted: 20 June 2018

Published online: 16 July 2018

\section{References}

1. Sellmyer MA, Desser TS, Maturen KE et al (2013) Physiologic, histologic, and imaging features of retained products of conception. RadioGraphics 33:781-796

2. Hamerlynck TWO, Blikkendaal MD, Schoot BC et al (2013) An alternative approach for removal of placental remnants : hysteroscopic morcellation. J Minim Invasive Gynecol 20:796-802. https://doi.org/10.1016/j.jmig.2013.04.024
3. Sadan O, Golan A, Girtler O et al (2004) Role of sonography in the diagnosis of retained products of conception. J Ultrasound Med 23:371-374

4. Pather S, Ford M, Reid R, Sykes P (2005) Postpartum curettage: an audit of 200 cases. Aust New Zeal J Obstet Gynaecol 45:368-371. https://doi.org/10. 1111/j.1479-828X.2005.00445.x

5. Dankert T, Vleugels M (2008) Hysteroscopic resection of retained placental tissue : a feasibility study. Gynecol Surg 5:121-124. https:/doi.org/10.1007/s10397-007-0354-x

6. Inal MM, Yildirim Y, Ertopcu K, Ozelmas I (2006) The predictors of retained products of conception following first-trimester pregnancy termination with manual vacuum aspiration. Eur J Contracept Reprod Heal Care 11:98-103. https://doi.org/10.1080/13625180500456742

7. Kitahara T, Sato Y, Kakui K et al (2011) Management of retained products of conception with marked vascularity. J Obstet Gynaecol Res 37:458-464. https://doi.org/10.1111/j.1447-0756.2010.01363.x

8. van den Bosch T, Daemen A, Van Schoubroeck D et al (2008) Occurrence and outcome of residual trophoblastic tissue: a prospective study. J Ultrasound Med 27:357-361

9. Vitner D, Filmer S, Goldstein I et al (2013) A comparison between ultrasonography and hysteroscopy in the diagnosis of uterine pathology. Eur J Obstet Gynecol Reprod Biol 171:143-145. https://doi.org/10.1016/j.ejogrb.2013.08.024

10. Hamerlynck TWO, van Vliet HAAM, Beerens AS et al (2016) Hysteroscopic morcellation versus loop resection for removal of placental remnants: a randomized trial. J Minim Invasive Gynecol 23:1172-1180. https://doi.org/10. 1016/j.jmig.2016.08.828

11. Goldenberg M, Schiff E, Achiron R, Lipitz S, Mashiach S (1997) Managing residual trophoblastic tissue: hysteroscopy for directing curettage. J Reprod Med 42:26-28

12. Cohen SB, Kalter-Ferber A, Weisz BS et al (2001) Hysteroscopy may be the method of choice for management of residual trophoblastic tissue. J Am Assoc Gynecol Laparosc 8:199-202

13. Faivre E, Deffieux X, Mrazguia C et al (2009) Hysteroscopic management of residual trophoblastic tissue and reproductive outcome: a pilot study. J Minim Invasive Gynecol 16:487-490 https://doi.org/10.1016/j.jmig.2009.04.011

14. Nicopoullos JDM, Treharne A, Raza A, Richardson R (2010) The use of a hysteroscopic resectoscope for repeat evacuation of retained products of conception procedures: a case series. Gynecol Surg 7:163-166

15. Golan A, Dishi M, Shalev A et al (2011) Operative hysteroscopy to remove retained products of conception: novel treatment of an old problem. J Minim Invasive Gynecol 18:100-103. https://doi.org/10.1016/j.jmig.2010.09.001

16. Rein DT, Schmidt T, Hess AP et al (2011) Hysteroscopic management of residual trophoblastic tissue is superior to ultrasound-guided curettage. J Minim Invasive Gynecol 18:774-778. https://doi.org/10.1016/j.jmig.2011.08.003

17. Yu D, Wong YM, Cheong Y et al (2008) Asherman syndrome-one century later. Fertil Steril 89:759-779. https://doi.org/10.1016/j.fertnstert.2008.02.096

18. Bosteels J, Weyers S, Kasius J et al (2015) Anti-adhesion therapy following operative hysteroscopy for treatment of female subfertility. Cochrane Database Syst Rev 11:CD011110. https://doi.org/10.1002/14651858.CD011110.pub2

19. Zegers-Hochschild F, Adamson GD, de Mouzon J et al (2009) International Committee for Monitoring Assisted Reproductive Technology (ICMART) and the World Health Organization (WHO) revised glossary of ART terminology, 2009*. Fertil Steril 92:1520-1524. https://doi.org/10.1016/j.fertnstert.2009.09.009

20. Higgins JPT, Green S (editors). Cochrane Handbook for Systematic Reviews of Interventions Version 5.1.0 [updated March 2011]. The Cochrane Collaboration, 2011. Available from www.handbook.cochrane.org

21. Review Manager 5 (RevMan 5) [Computer program]. Version 5.3. Copenhagen: Nordic Cochrane Centre, The Cochrane Collaboration, 2014

22. Fuchs N, Smorgick N, Ben Ami I et al (2014) Intercoat (Oxiplex/AP gel) for preventing intrauterine adhesions after operative hysteroscopy for suspected retained products of conception: double-blind, prospective, randomized pilot study. J Minim Invasive Gynecol 21:126-130. https://doi. org/10.1016/j.jmig.2013.07.019

23. Smorgick N, Barel O, Fuchs N et al (2014) Hysteroscopic management of retained products of conception: meta-analysis and literature review. Eur J Obstet Gynecol Reprod Biol 173:19-22. https://doi.org/10.1016/j.ejogrb.2013.11.020

24. Hooker AB, Aydin H, Brölman HAM, Huirne JAF (2016) Long-term complications and reproductive outcome after the management of retained products of conception : a systematic review. Fertil Steril 105:156-164. https://doi.org/10.1016/j.fertnstert.2015.09.021 